\title{
Simple and Accurate Analytical Solutions of the Electrostatically Actuated Curled Beam Problem
}

\author{
Mohammad I. Younis ${ }^{1,2}$ \\ 1. Department of Mechanical \\ Engineering \\ State University of New York \\ Binghamton, NY, USA \\ myounis@binghamton.edu \\ 2. Physical Sciences and \\ Engineering Division \\ King Abdullah University of \\ Science and Technology \\ (KAUST), Thuwal 23955-6900, \\ Saudi Arabia \\ mohammad.younis@kaust.edu.
}

\begin{abstract}
We present analytical solutions of the electrostatically actuated initially deformed cantilever beam problem. We use a continuous Euler-Bernoulli beam model combined with a single-mode Galerkin approximation. We derive simple analytical expressions for two commonly observed deformed beams configurations: the curled and tilted configurations. The derived analytical formulas are validated by comparing their results to experimental data in the literature and numerical results of a multi-mode reduced order model. The derived expressions do not involve any complicated integrals or complex terms and can be conveniently used by designers for quick, yet accurate, estimations. The formulas are found to yield accurate results for most commonly encountered microbeams of initial tip deflections of few microns. For largely deformed beams, we found that these formulas yield less accurate results due to the limitations of the single-mode approximations they are based on. In such cases, multi-mode reduced order models need to be utilized.
\end{abstract}

\section{INTRODUCTION}

Cantilever microbeams are commonly fabricated with unavoidable initial tilt or curling due to stress gradients and other imperfections [1,2]. Despite the low level of this initial deflection of the beam profile compared to its length; it has significant effect on its static and dynamic behavior. This is even more critical in the case of electrostatic excitation and capacitive detection, which have strong dependence on the gap separating the cantilever beam from the substrate or lower electrode. The gap width $d$ is typically one to two microns, which is very much close to the maximum tip deflection of most curled cantilever microbeams. The impact of this is that many of the simple analytical techniques and formula that have been developed for straight cantilever microbeams are no longer valid. For instance, the below well-known formula of the pull-in voltage $V_{\text {pull }}$, which was developed based on a spring mass model, does not apply

$$
V_{p u l l}=\sqrt{\frac{8 k d^{3}}{27 \varepsilon A}}
$$

where $k$ is the effective stiffness of the cantilever microbeam for a uniformly distributed load [3], $\varepsilon$ is the dielectric constant of the gap medium, and $A$ is the electrode area. In this formula, there is no rule of choosing an appropriate $d$ that will lead to accurate prediction (whether measuring from the tip of the cantilever to the ground or ignoring the curling and measuring from the straight position of the beam; all lead to erroneous predictions.) The influence of the curling also includes wrong modeling of squeeze-film damping (strongly depends on the gap width), pull-in time, natural frequencies, and bouncing effects of the interaction of the cantilever tip with the substrate. Several studies have recognized the importance of accounting for the curling of cantilever beams on its behavior, and especially on pull-in calculations. Attempts to develop analytical formula to predict the pull-in voltage of curled microbeams have been made. These studies were motivated by the need to accurately predict pull-in and at the same time develop handy and practical analytical formula, which designers can conveniently use. Among the recent contributions, Legtenberg et al. [4] presented the design and 
performance of an electrostatic actuator consisting of a laterally compliant cantilever beam and a fixed curved electrode. They derived pull-in formula using the Rayleigh-Ritz method. Wei et al. [5] solved for the static deflection of a cantilever beam subjected to a point load at the tip, which was related to the electrostatic force of the initially deflected beam. Then, by calculating the beam deflection due to this point load and imposing a pull-in condition that pull-in occurs when the beam has deflected half way through the entire gap, a pull-in formula was derived. Lishchynska et al. [6] modeled the electrostatic behavior of micromachined cantilevers incorporating residual stress gradient and non-ideal anchors using finite-element simulation data and a lumped-parameter model.

$\mathrm{Hu}$ [7] presented three analytical models of micro curled beams subjected to electrostatic loads, varying in the way the electrostatic loads are truncated in a Taylor's series, which are the full-order, the fourth-order and the third-order models. They derived for each the corresponding closed form solutions for the pull-in voltages based on the Euler-Bernoulli beam theory and an energy method. Hu and Wei [8] improved on the model of [7] by including the fringing effect of the electrostatic field between the beam and the substrate. Chuang et al. [9] further improved the model in [7] and [8] by modeling the non-ideal support through including a torsional spring at the anchor in the model.

Saha et al. [10] presented a model for the spring constant and the pull-down voltage of a non-uniform RF MEMS curled cantilever under electrostatic actuation. Results were validated by comparison with a finite element model. Hu et al. [11] investigated the pull-in behavior of electrostatically actuated microbeams with large initial curved configuration, including both cantilever and clamped-clamped beams. The theoretical formulation is based on an arc-coordinate system. The nonlinear governing equations are solved using the differential quadrature method (DQM) and an iterative algorithm. Vaishali et al. [12] used an analytical model based on a lumped parameter model to determine the shift in the resonant frequency of curled cantilever beams under dc bias voltages. Abdulla et al. [13] presented modeling, fabrication and measurements of curled micro cantilevers. A Galerkin-based static model is used to predict the pull-in voltages. A dynamic model is used to investigate the shift in resonance frequency by the electrostatic spring softening effect. Results are validated by comparing to experimental data. Ou et al. [14] derived a semi-analytical formulation for computing the pull-in voltage of a curled cantilever beam by assuming an admissible deformation shape and using the energy method to determine the coefficients of the shape functions.

It is noted that, despite the progress that has been achieved into modeling and predicting the pull-in voltage of curled cantilever beams and the several proposed formulas; all of these require evaluating complex integrals, are not easy to use, and involve complicated calculations. It would be of great advantage to
MEMS designers to be able to use simple formula that can be programmed on calculators and that do not require intensive calculations. Such analytical expressions can be considered handy, pure analytical, and practical. In this paper, we aim to present such formulas.

The organization of the paper is as follows. First, we present the problem formulation based on an Euler-Bernoulli beam model. Then, the Galerkin procedure is applied. Using one mode in the Galerkin procedure, a single-degree-of-freedom model governing the dynamic behavior of the cantilever beam tip is derived. Based on this model and the bifurcation criteria of the pull-in, and assuming parabolic shape of the cantilever beam, simple analytical formulas of the pull-in voltage and the corresponding maximum deflection of a curled beam are derived. A similar procedure is repeated assuming a rigidly tilted cantilever beam, which is the case of beams of non-ideal (flexible) supports. The results of the formulas are compared against the experimental data in the literature as well as the multi-mode reduced-order model results. Finally, the case of large curling of beams is considered, and based on which, criteria for the accuracy of the extracted analytical formulas are deduced.

\section{PROBLEM MODEL}

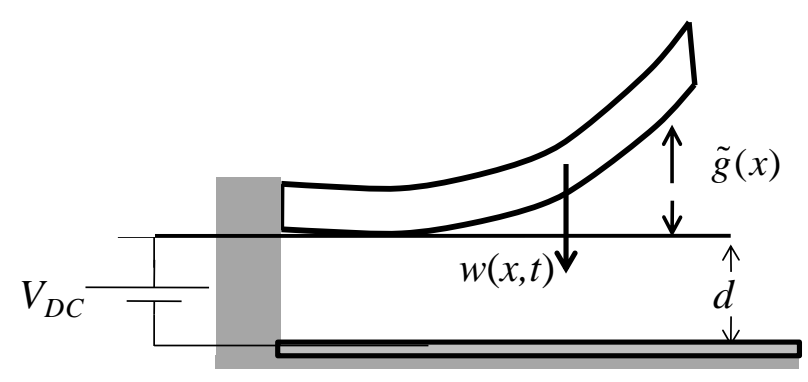

Fig. 1. Schematic of an initially deformed electrostatically actuated cantilever microbeam.

We consider an electrostatically actuated cantilever microbeam of length $l$, thickness $h$, and width $b$. The beam anchor is raised above the substrate a distance $d$ while the beam itself is curled above the anchor level with a profile $\tilde{g}(x)$, where $x$ is the position along the beam length, as shown in Fig. 1. The equation of motion governing the deflection $w(x, t)$ of the microbeam in space $x$ and time $t$ can be written as

$$
E I w^{\prime \prime \prime \prime}+c \dot{w}+\rho b h \ddot{w}=\frac{\varepsilon b V_{D C}^{2}}{2(d-w+\tilde{g})^{2}}
$$

where $I$ is the moment of inertia of the cross section, $E$ is Young's modulus, $\rho$ is the material density, $\mathrm{c}$ is a viscous damping coefficient, $V_{D C}$ is the polarization voltage, and $\varepsilon$ is 
the dielectric constant of the gap medium. Above, the superscript prim indicates spatial derivative and the dot indicates time derivative.

For convenience, Eq. (2) is normalized. Toward this, the following nondimensional variables (denoted by hats) are introduced

$$
\hat{w}=\frac{w}{d}, \quad \hat{x}=\frac{x}{l}, \quad \hat{t}=\frac{t}{T}
$$

where $T$ is a time scale defined as

$$
T=\sqrt{\frac{\rho b h l^{4}}{E I}}
$$

Substituting Eq. (3) into Eq. (2) and dropping the hats from the nondimensional variables for convenience, the following nondimensional equation is derived:

$$
\frac{\partial^{4} w}{\partial x^{4}}+\frac{\partial^{2} w}{\partial t^{2}}+c_{n o n} \frac{\partial w}{\partial t}=\frac{\alpha_{2} V_{D C}^{2}}{(1-w+g)^{2}}
$$

where $g(x)$ is the nondimensional beam profile. The parameters appearing in Eq. (5) are defined as

$$
\alpha_{2}=\frac{6 \varepsilon l^{4}}{E h^{3} d^{3}} ; \quad c_{n o n}=\frac{12 c l^{4}}{E T b h^{3}}
$$

The boundary conditions of Eq. (2), and hence Eq. (5), are zero slope and displacement at $x=0$ and zero shear force and moment at $x=l$, that is

$$
w(0, t)=w^{\prime}(0, t)=0 ; \quad w^{\prime \prime}(l, t)=w^{\prime \prime \prime}(l, t)=0 ;
$$

Next, we generate a reduced-order model ROM by discretizing equations (5) into a finite-degree-of-freedom system consisting of ordinary-differential equations in time using the Galerkin method. In order to deal with the complicated electrostatic force term, we multiply the whole equation by the denominator of the electrostatic force term $[3,15,16]$

$$
(1-w+g)^{2} \frac{\partial^{4} w}{\partial x^{4}}+(1-w+g)^{2} \frac{\partial^{2} w}{\partial t^{2}}+(1-w+g)^{2} c_{n o n} \frac{\partial w}{\partial t}=\alpha_{2} V_{D C}^{2}
$$

We use the undamped linear mode shapes of the straight (unactuated) microbeam as basis functions in the Galerkin procedure. To this end, we express the deflection as

$$
w(x, t)=\sum_{i=1}^{n} u_{i}(t) \phi_{i}(x)
$$

where $u_{i}(t)$ is the $i$ th generalized coordinate and $\phi_{i}(x)$ is the $i$ th linear undamped mode shape of the straight microbeam governed by

$$
\phi_{i}^{\prime \prime \prime \prime}-\omega_{\text {non,i }}^{2} \phi_{i}=0
$$

and the corresponding boundary conditions according to Eq. (7). In Eq.(10), $\omega_{n o n, i}$ refers to the $i^{\text {th }}$ non-dimensional natural frequency. The mode shapes are normalized such that $\int_{0}^{1} \phi_{i}^{2} d x=1$.

Subsequently, Eq. (9) is substituted into Eq. (8), $\phi_{i}^{\prime \prime \prime \prime}$ is replaced with $N_{n o n} \phi_{i}^{\prime \prime}+\omega_{n o n, i}^{2} \phi_{i}$, the outcome is then multiplied by the modeshape $\phi_{j}$ and is integrated over the beam domain from 0-1

$$
\begin{aligned}
& \int_{0}^{1} \phi_{j}\left(1-\sum_{l=1}^{n} u_{l} \phi_{l}\right)^{2}\left(\sum_{i=1}^{n} u_{i} \omega_{n o n, i}^{2} \phi_{i}+\sum_{i=1}^{n} \ddot{u}_{i} \phi_{i}\right) d x+ \\
& c_{n o n} \int_{0}^{1} \phi_{j}\left(1-\sum_{l=1}^{n} u_{l} \phi_{l}\right)^{2}\left(\sum_{i=1}^{n} \dot{u}_{i} \phi_{i}\right) d x=\alpha_{2} V_{D C}^{2} \int_{0}^{1} \phi_{j} d x
\end{aligned}
$$

Equation (11) represents a multi-mode ROM. Executing the spatial integrals and applying the orthogonality condition of the modeshape yield an $n$ system of ordinary differential equations, which can be integrated numerically in time for dynamic simulations. For static simulations, the time varying modal coordinates are replaced with constant coefficients yielding nonlinear algebraic equations to be solved for.

Using one term, $n=1$, in the Galerkin expansion and rearranging reduce the equation to

$$
\begin{aligned}
& {\left[\ddot{u}_{1}+c_{n o n} \dot{u}_{1}+\omega_{n o n}^{2} u_{1}\right] \int_{0}^{1} \phi\left\{\left(\phi+2 g \phi+g^{2} \phi-2 u_{1} \phi^{2}-2 g u_{1} \phi^{2}+u_{1}^{2} \phi^{3}\right)\right\} d x} \\
& =\alpha_{2} V_{D C}^{2} \int_{0}^{1} \phi_{j} d x
\end{aligned}
$$

To proceed further, a specific shape of the beam initial profile needs to be implemented. We discuss here two initial shapes, which are commonly observed experimentally: the parabolic and the linear shapes [2]. Which one to be used practically requires optical inspection of the fabricated microbeams to determine the best approximation of their profile, and hence the appropriate formula to implement of the next derived ones.

\section{ANALYTICAL DERIVATIONS}

\subsection{The Parabolic Shape}

Cantilever microbeams can be initially curled due to stress gradient, which is equivalent to an applied moment on the beam, or can be tilted due to the flexible anchor [1-3]. The stress gradient $\Gamma$ results in a parabolic shape of the beam, which can be expressed as [3]

$$
g(x)=\frac{\Gamma l}{2 E} x^{2}
$$

A flexible anchor on the other hand results in a rigid-body tilt of a linear shape [2,3]. First, we will assume the beam to be 
deflected purely due to the stress gradient. In the next subsection we will derive the formula for the tilted case. To proceed, we assume the initial shape of the beam expressed generally as

$$
g(x)=\gamma x^{2}
$$

where $\gamma=\frac{W_{0}^{t i p}}{d}$. Equation (14) is equivalent to Eq. (13), but expressed conveniently in terms of $W_{0}^{t i p}$, the maximum tip deflection of the cantilever beam, which can be measured using an optical profiler or interferometer.

Next, we recall Eq.(9) and express the beam tip deflection $W$ as

$$
W(t)=w(1, t)=u_{1}(t) \phi(1)=2 u_{1}(t)
$$

Hence, substituting Eq. (14) into Eq. (12), expressing the equation in terms of $W$ according to Eq. (13), executing the integrals, and re-arranging yield

$$
\begin{aligned}
& \ddot{W}+c_{n o n} \dot{W}+\omega_{\text {non }}^{2} W= \\
& \frac{1.57 \alpha_{2} V_{D C}^{2}}{1+1.35 \gamma+0.51 \gamma^{2}-(1.48+1.1 \gamma) W+0.59 W^{2}}
\end{aligned}
$$

Equation (16) can be viewed as an equivalent spring-massdamper model, which takes into account the spatial shape of the beam as well as its initial tilt. The accuracy of the model for dynamic behavior has not been investigated yet however, and hence care should be taken when adopting this equation. Also, one should recall that this is a single mode model, which has its limitation in terms of accuracy and what it can capture. Having said that, the model still represents an improvement over the classical spring-mass-damper system derived based on effective stiffness, inertia, and damper, which does not account for any initial tilt or shape factors.

Next, we proceed with the static analysis. Thus, we set $c_{\text {non }}=0$ and assume $W$ time independent, which yields

$$
\begin{aligned}
& \omega_{\text {non }}^{2} W=F= \\
& \frac{1.57 \alpha_{2} V_{D C}^{2}}{1+1.35 \gamma+0.51 \gamma^{2}-(1.48+1.1 \gamma) W+0.59 W^{2}}
\end{aligned}
$$

Equation (17) represents the equilibrium equation. Solving it yields three solutions, two of them are physical and one nonphysical predicting deflection into the substrate. The stability of the two physical solutions can be determined by solving the eigenvalue problem associate with the system Jacobian [3]. This yields the following expression of the eigenvalues $\lambda$ :

$$
\lambda=\partial_{W} F-\omega_{\text {non }}^{2}
$$

As a byproduct, the square root of the real part of the eigenvalue yields an expression for the natural frequency of the curled beam as it varies with $V_{D C}$

$$
\sqrt{\omega_{d c}=} \sqrt{\omega_{n o n}^{2}+\frac{1.57 \alpha_{2} V_{D C}^{2}(-1.48+1.17 W-1.1 \gamma)}{\left[1+1.35 \gamma+0.51 \gamma^{2}-(1.48+1.1 \gamma) W+0.59 W^{2}\right]^{2}}}
$$

In Eq. (19), $W$ refers to the static deflection at the considered $V_{D C}$.

To derive an analytic expression for the pull-in voltage $V_{\text {pull }}$, we set the eigenvalue $=0$

$$
\begin{aligned}
& \omega_{n o n}^{2}+\frac{1.57 \alpha_{2} V_{D C}^{2}(-1.48+1.17 W-1.1 \gamma)}{\left[1+1.35 \gamma+0.51 \gamma^{2}-(1.48+1.1 \gamma) W+0.59 W^{2}\right]^{2}} \\
& =0
\end{aligned}
$$

Solving Eq.(17) and Eq.(20) simultaneously yields the following expressions for the maximum tip deflection of the cantilever at pull-in $W_{p}$ and the corresponding voltage $V_{\text {pull }}$ :

$$
\begin{aligned}
& W_{p}=0.84+0.62 \gamma-0.31 \sqrt{(0.65+\gamma)(2.23+\gamma)} \\
& V_{\text {pull }}= \\
& \sqrt{\left[7.9 W_{p}\left(1 .+0.59 W_{p}^{2}+W_{p}(-1.48-1.1 \gamma)+1.35 \gamma+0.51 \gamma^{2}\right)\right] /} \alpha_{2}
\end{aligned}
$$

Equations (21) and (22) are two simple analytical expressions that can be evaluated using a simple calculator. They do not involve any complicated integrals.

It is interesting to note that for the special case of no curling, $\gamma$ $=0$, which reduces the equations to

$$
W_{p}=0.47 ; \quad \alpha_{2} V_{\text {pull }}^{2}=1.614
$$

These values are close to those observed when using a multimode Galerkin procedure and numerical solutions, where it was noted that $\alpha_{2} V_{\text {pull }}^{2} \approx 1.72$ [3].

\subsection{The Tilted Shape}

In this case, the beam initial curvature, due to non-ideal support, looks like a linear profile rather than a curvy parabolic shape. Hence, the initial shape is taken as

$$
g(x)=\gamma x
$$

where also here $\gamma=\frac{W_{0}^{t i p}}{d}$. Following a similar procedure as in the previous section yields the below single-degree-of-freedom model

$$
\begin{aligned}
& \ddot{W}+c_{\text {non }} \dot{W}+\omega_{\text {non }}^{2} W= \\
& \frac{1.57 \alpha_{2}\left[V_{D C}+V_{A C} \cos (\Omega t)\right]^{2}}{1+1.613 \gamma+0.675 \gamma^{2}-(1.48+1.255 \gamma) W+0.59 W^{2}}
\end{aligned}
$$


Also, following similar stability steps as in Section 2.1 yields the following expressions for the maximum tip deflection of the cantilever at pull-in $W_{p}$ and the corresponding voltage $V_{\text {pull }}$ :

$$
W_{P}=0.84+0.71 \gamma-0.35 \sqrt{(0.71+\gamma)(1.53+\gamma)}
$$

$V_{\text {Pull }}=$

$$
\sqrt{\left[7.9 W_{p}\left(1 .+0.59 W_{p}^{2}+W_{p}(-1.48-1.26 \gamma)+1.61 \gamma+0.675 \gamma^{2}\right)\right]} / \alpha_{2}
$$

\section{RESULTS}

In this section, the validity of the derived formulas of Eq.(22) and Eq.(27) is demonstrated by comparison with the experimental data of [17]. These data represent a comprehensive experimental study of initially deformed cantilever beams of lengths ranging from 100-500 $\mu \mathrm{m}$. They are commonly used in the literature for comparison purposes with the modeling results. Table 1 below lists the material and geometric properties of the beams array. Note here that all the beams share the same stress gradient, and hence, the same radius of curvature $R$. Table 2 lists the beam lengths and their maximum tip deflection, as calculated based on the provided radius of curvature ( $W_{0}^{t i p}=R(1-\cos (l / R))$, in addition to their measured pull-in voltage [17].

Before we show results using the new model of the curved beams, it is worth to compare the reported experimental data of [17] with the theoretical results assuming a straight beam, and using a beam model. Figure 2 a depicts the comparison whereas Fig. $2 b$ shows the estimated error. For small beams the initial deflection is too small to make any difference, but as the beam length increases, the initial deflection increases, and so does the error. It is clear that using a straight beam theory for pull-in calculations of initially deformed beams leads to severely wrong results.

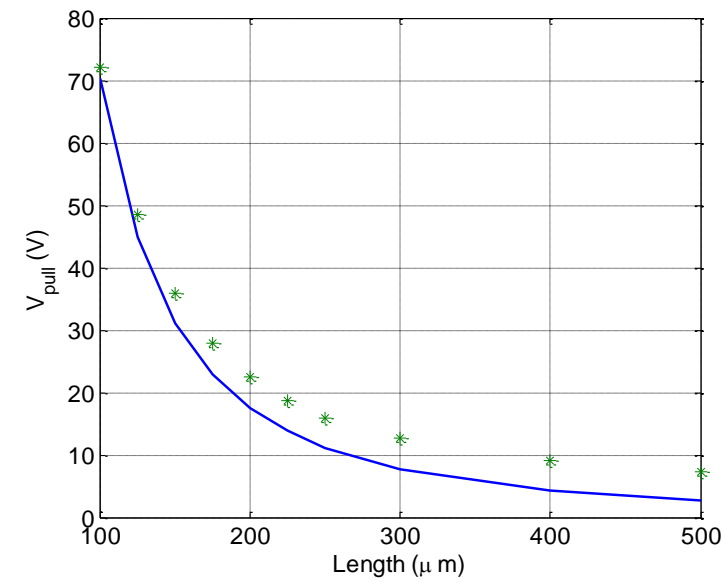

(a)

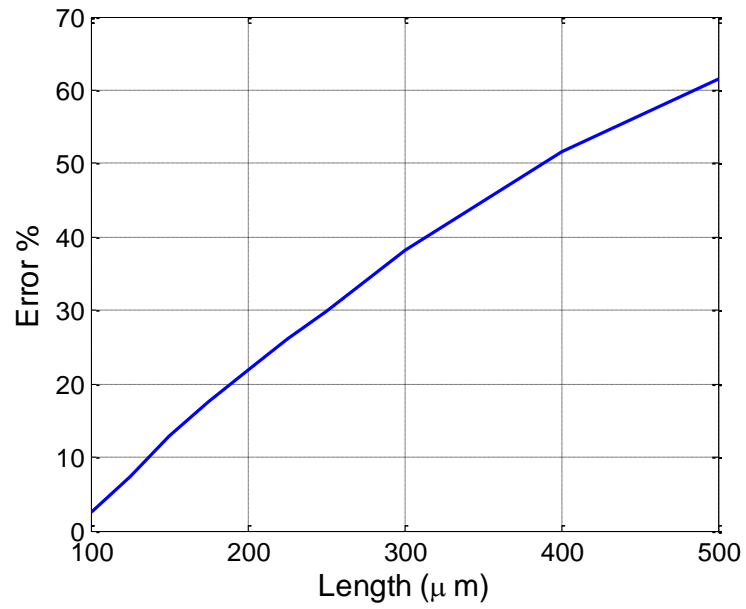

(b)

Figure 2: (a) Pull-in voltage of a curled beam calculated using a straight beam theory (solid) versus the experimental data of

[17]. (b) The calculated percentage error.

Table 1. Material and geometrical parameters of the polysilicon curled beams of [17].

\begin{tabular}{|l|l|}
\hline Parameter & Value \\
\hline Young's modulus, $E$ & $153 \mathrm{GPa}$ \\
\hline $\begin{array}{l}\text { Relative dielectric constant between the beam } \\
\text { and the substrate } \varepsilon_{\mathrm{r}}\end{array}$ & 1.2046 \\
\hline Beam length, $L$ & $100-500 \mu \mathrm{m}$ \\
\hline Beam width, $b$ & $40 \mu \mathrm{m}$ \\
\hline Beam thickness, $h$ & $2.1 \mu \mathrm{m}$ \\
\hline Initial radius of curvature, $R$ & $40000 \mu \mathrm{m}$ \\
\hline Initial gap, $d$ & $2.4 \mu \mathrm{m}$ \\
\hline
\end{tabular}

Table 2. The length of the microbeams of [17], their maximum tip deflection, and their measured pull-in voltages.

\begin{tabular}{|l|l|l|}
\hline Length $(\mu \mathrm{m})$ & $W_{0}^{\text {tip }}(\mu \mathrm{m})$ & Measured Pull-in [17] \\
\hline 100 & 0.125 & 72.07 \\
\hline 125 & 0.195 & 48.6 \\
\hline 150 & 0.28 & 35.82 \\
\hline 175 & 0.38 & 27.89 \\
\hline 200 & 0.5 & 22.55 \\
\hline 225 & 0.63 & 18.79 \\
\hline 250 & 0.78 & 15.95 \\
\hline 300 & 1.12 & 12.61 \\
\hline 400 & 2 & 9.10 \\
\hline 500 & 3.12 & 7.27 \\
\hline
\end{tabular}


Next, we apply both the curled and tilted formulas, Eq.(22) and Eq.(27), on the beams of Table 2 to determine their pull-in voltage. The results as compared to the experimental measurements of [17] are depicted in Fig.3. As seen, both formulas predict results that are very close to the experimental data. In addition, we calculated the pull-in of these beams using a 4-mode reduced order model of Eq. (11) when implementing both the curled and tilted configurations. The results of the ROM and the analytical formulas, the estimated errors as compared to the experimental data, and the theoretical results of [5] and [7] and their estimated errors are all presented in Table 3. It is noted that the mean percentage error of both the curled and tilted formulas are less than $5 \%$, which is considered very low. The multi-mode ROM in this particular set of data has not shown much improvement in accuracy. The tilted model here shows more accurate results than the curled model, which may be due to the fact that these beams are not purely curled and might have some flexibility at their edges. However this cannot be known for sure unless accurate profiles of the beams are captured using interferometers or SEM pictures. In general it is expected that, in the case of uncertain initial deformation profile, both formulas will capture the proper range of the actual data.

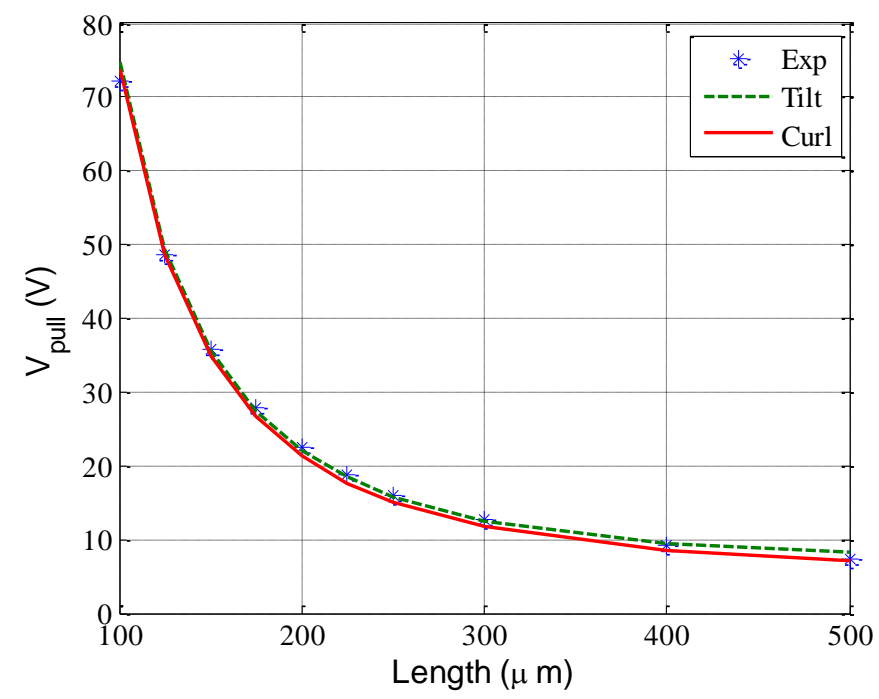

Figure 3: Comparison of the experimental data of [17] against the theoretical predictions using the tilted and curled beam models.

Table 3. Comparison of the calculated pull-in voltage using various analytical models from the literature, the two developed formulas (tilted and curled), and the multi-mode reduced order model against the experimental data of [17]. Shown also in parentheses are the estimated percentage errors.

\begin{tabular}{|c|c|c|c|c|c|c|c|c|c|}
\hline \multirow[b]{2}{*}{$\begin{array}{l}\text { Length } \\
(\mu \mathrm{m})\end{array}$} & \multirow[b]{2}{*}{$\begin{array}{l}W_{0}^{t i p} \\
(\mu \mathrm{m})\end{array}$} & \multicolumn{8}{|c|}{$\begin{array}{l}\text { Pull-in Voltage (V) } \\
\text { (Error \%) }\end{array}$} \\
\hline & & $\begin{array}{l}\text { Full } \\
\text { Order }\end{array}$ & $\begin{array}{l}\text { Third } \\
\text { Order }\end{array}$ & $\begin{array}{l}\text { Wei's } \\
\text { model }\end{array}$ & $\begin{array}{l}\text { Curled } \\
\text { Model }\end{array}$ & $\begin{array}{l}\text { Tilted } \\
\text { Model }\end{array}$ & $\begin{array}{l}\text { ROM } \\
\text { (4md) } \\
\text { Cur }\end{array}$ & $\begin{array}{l}\text { ROM } \\
\text { (4md) } \\
\text { Tilt }\end{array}$ & $\begin{array}{l}\text { Measure } \\
{[17]}\end{array}$ \\
\hline 100 & 0.125 & $\begin{array}{l}75.51 \\
(9.25 \%)\end{array}$ & $\begin{array}{l}78.74 \\
(18.81 \%)\end{array}$ & $\begin{array}{l}81.09 \\
(12.51 \%)\end{array}$ & $\begin{array}{l}73.86 \\
(2.5 \%)\end{array}$ & $\begin{array}{l}74.65 \\
(3.58 \%)\end{array}$ & $\begin{array}{l}75.64 \\
(4.95 \%)\end{array}$ & $\begin{array}{l}76.48 \\
(6.12 \%)\end{array}$ & 72.07 \\
\hline 125 & 0.195 & $\begin{array}{l}49.67 \\
(2.21 \%)\end{array}$ & $\begin{array}{l}56.35 \\
(15.95 \%)\end{array}$ & $\begin{array}{l}66.72 \\
(37.29 \%)\end{array}$ & $\begin{array}{l}48.58 \\
(0.048 \%)\end{array}$ & $\begin{array}{l}49.37 \\
(1.59 \%)\end{array}$ & $\begin{array}{l}49.8 \\
(2.47 \%)\end{array}$ & $\begin{array}{l}50.54 \\
(3.99 \%)\end{array}$ & 48.6 \\
\hline 150 & 0.28 & $\begin{array}{l}35.65 \\
(0.48 \%)\end{array}$ & $\begin{array}{l}40.46 \\
(12.93 \%)\end{array}$ & $\begin{array}{l}48.18 \\
(34.50 \%)\end{array}$ & $\begin{array}{l}34.86 \\
(2.69 \%)\end{array}$ & $\begin{array}{l}35.66 \\
(0.45 \%)\end{array}$ & $\begin{array}{l}35.76 \\
(0.17 \%)\end{array}$ & $\begin{array}{l}36.52 \\
(1.95 \%)\end{array}$ & 35.82 \\
\hline 175 & 0.38 & $\begin{array}{l}27.25 \\
(2.31 \%)\end{array}$ & $\begin{array}{l}30.87 \\
(10.69 \%)\end{array}$ & $\begin{array}{l}36.74 \\
(31.74 \%)\end{array}$ & $\begin{array}{l}26.60 \\
(4.62 \%)\end{array}$ & $\begin{array}{l}27.40 \\
(1.73 \%)\end{array}$ & $\begin{array}{l}27.3 \\
(2.11 \%)\end{array}$ & $\begin{array}{l}28.1 \\
(0.75 \%)\end{array}$ & 27.89 \\
\hline 200 & 0.5 & $\begin{array}{l}21.76 \\
(3.48 \%)\end{array}$ & $\begin{array}{l}24.65 \\
(9.34 \%)\end{array}$ & $\begin{array}{l}29.48 \\
(30.74 \%)\end{array}$ & $\begin{array}{l}21.26 \\
(5.73 \%)\end{array}$ & $\begin{array}{l}22.01 \\
(2.12 \%)\end{array}$ & $\begin{array}{l}21.8 \\
(3.3 \%)\end{array}$ & $\begin{array}{l}22.62 \\
(0.31 \%)\end{array}$ & 22.55 \\
\hline 225 & 0.63 & $\begin{array}{l}18.01 \\
(4.17 \%)\end{array}$ & $\begin{array}{l}20.39 \\
(8.52 \%)\end{array}$ & $\begin{array}{l}24.30 \\
(29.33 \%)\end{array}$ & $\begin{array}{l}17.61 \\
(6.28 \%)\end{array}$ & $\begin{array}{l}18.43 \\
(1.91 \%)\end{array}$ & $\begin{array}{l}18.04 \\
(3.99 \%)\end{array}$ & $\begin{array}{l}18.9 \\
(0.59 \%)\end{array}$ & 18.79 \\
\hline 250 & 0.78 & $\begin{array}{l}15.30 \\
(4.07 \%)\end{array}$ & $\begin{array}{l}17.34 \\
(8.74 \%)\end{array}$ & $\begin{array}{l}20.79 \\
(30.37 \%)\end{array}$ & $\begin{array}{l}15.0 \\
(5.85 \%)\end{array}$ & $\begin{array}{l}15.85 \\
(0.64 \%)\end{array}$ & $\begin{array}{l}15.36 \\
(3.7 \%)\end{array}$ & $\begin{array}{l}16.24 \\
(1.82 \%)\end{array}$ & 15.95 \\
\hline 300 & 1.12 & $\begin{array}{l}11.82 \\
(6.24 \%)\end{array}$ & $\begin{array}{l}13.38 \\
(6.10 \%)\end{array}$ & $\begin{array}{l}16.12 \\
(27.81 \%)\end{array}$ & $\begin{array}{l}11.68 \\
(7.37 \%)\end{array}$ & $\begin{array}{l}12.53 \\
(0.61 \%)\end{array}$ & $\begin{array}{l}11.84 \\
(6.1 \%)\end{array}$ & $\begin{array}{l}12.8 \\
(1.5 \%)\end{array}$ & 12.61 \\
\hline 400 & 2 & $\begin{array}{l}8.36 \\
(8.14 \%)\end{array}$ & $\begin{array}{l}9.45 \\
(3.82 \%)\end{array}$ & $\begin{array}{l}11.69 \\
(28.44 \%)\end{array}$ & $\begin{array}{l}8.50 \\
(6.56 \%)\end{array}$ & $\begin{array}{l}9.40 \\
(3.39 \%)\end{array}$ & $\begin{array}{l}8.36 \\
(8.13 \%)\end{array}$ & $\begin{array}{l}9.52 \\
(4.6 \%)\end{array}$ & 9.10 \\
\hline 500 & 3.12 & $\begin{array}{l}6.77 \\
(6.78 \%)\end{array}$ & $\begin{array}{l}7.65 \\
(5.31 \%)\end{array}$ & $\begin{array}{l}9.69 \\
(33.33 \%)\end{array}$ & $\begin{array}{l}7.20 \\
(0.90 \%)\end{array}$ & $\begin{array}{l}8.17 \\
(12.42 \%)\end{array}$ & $\begin{array}{l}6.8 \\
(6.46 \%)\end{array}$ & $\begin{array}{l}8.1 \\
(11.4 \%)\end{array}$ & 7.27 \\
\hline $\begin{array}{l}\text { Mean } \\
\text { rror }\end{array}$ & & $4.27 \%$ & $10.02 \%$ & $29.61 \%$ & $4.25 \%$ & $2.84 \%$ & $4.14 \%$ & $3.31 \%$ & \\
\hline
\end{tabular}

Despite the excellent accuracy of the developed formulas demonstrated in the previous section, one should recall that these are based on a single-mode approximation. Therefore, it is expected that these formulas are limited in their accuracy for small initial deformations, as in the case of the beams of Table 2. For instance, it was found previously that multiple modes are needed to capture accurately the pull-in instability under mechanical shock and that a single mode prediction is inaccurate [18]. Similar conclusion was drawn for the pull-in voltage of initially curved clamped-clamped microbeams (arches) [19]. Based on this, we examine the limitation of the developed formulas by studying largely initially deflected beams. As case studies, we investigate the beams of $\mathrm{Hu}$ and Wei [8] of properties listed in Table 4. These beams are largely deformed, as indicated in the second column of Table 5. It was indicated clearly in [8] that these beams are curled and not tilted, according to the pictures shown in [8]. We calculated the pull-in voltage of these beams using the analytical formulas of the tilted and curled profiles as well as using the ROM assuming 4 modes. The results as compared to the experimental data are presented in Table 5. It is seen clearly that in this regime, the tilted and curled results differ significantly. Moreover, the formulas results differ also significantly from the ROM results. The ROM of the curled beam are close to the experimental data. The conclusion of this table is that the developed formulas do not yield correct results in the cases of largely deformed beams; this is due to the fact that a singlemode approximation breaks down in this regime. To confirm our conclusion about the convergence of the model, we calculate the pull-in voltage of the beams using 1-4 mode ROM, Table 6.

Finally, we adopt the $600 \mu \mathrm{m}$ of Table 6 , as a case study, and investigate its pull-in voltage as calculated using 1-4 modes of the ROM, while its maximum tip deflection varies from very small values to large values, Fig. 4. The figure indicates that using 3 and 4 modes yields close and converged results for most of the tip-deflection range. Using the analytical formula, 
or the 1-mode ROM, is accurate only up to a maximum tip deflection of $5 \mu \mathrm{m}$. Hence, this establishes the range of validity of the developed analytical formulas.

Table 4. The material and geometrical parameters of the curled aluminum beam of [8].

\begin{tabular}{|c|c|}
\hline Parameter & Value \\
\hline Young's modulus, $E(\mathrm{GPa})$ & 74.14 \\
\hline Beam length, $l(\mu \mathrm{m})$ & $400,500,600$ \\
\hline Initial radius of curvature, $\rho(\mu \mathrm{m})$ & 2781,3641, \\
& 4359 \\
\hline Beam width, $b(\mu \mathrm{m})$ & 50 \\
\hline Beam thickness, $h(\mu \mathrm{m})$ & 1.32 \\
\hline Initial gap, $d(\mu \mathrm{m})$ & 1.63 \\
\hline $\begin{array}{c}\text { Relative dielectric constant between the beam } \\
\text { and the ground }\end{array}$ & 1 \\
\hline
\end{tabular}

Table 5. Pull-in voltage of the beams of [8] calculated using various approaches.

\begin{tabular}{|l|l|l|l|l|l|l|}
\hline $\begin{array}{l}\text { Length } \\
(\mu \mathrm{m})\end{array}$ & $\begin{array}{l}W_{0}^{\text {tip }} \\
(\mu \mathrm{m})\end{array}$ & $\begin{array}{l}\text { Analytical } \\
\text { Curled }\end{array}$ & $\begin{array}{l}\text { Analytical } \\
\text { Tilted }\end{array}$ & $\begin{array}{l}\text { ROM } \\
(4 \mathrm{md}) \\
\text { Tilt }\end{array}$ & $\begin{array}{l}\text { ROM } \\
(4 \mathrm{md}) \\
\text { cur }\end{array}$ & $\begin{array}{l}\text { Exp } \\
{[8]}\end{array}$ \\
\hline 400 & 28.7 & 45 & 55.47 & 46.96 & 19.78 & 20.25 \\
\hline 500 & 34.28 & 37 & 45.6 & 38.28 & 15.4 & 15.63 \\
\hline 600 & 41.23 & 33.43 & 41.2 & 34.36 & 13.18 & 13.38 \\
\hline
\end{tabular}

Table 6. Pull-in voltage of the beams of [8] calculated using 1, 2,3 , and 4 ROM of the curled configuration.

\begin{tabular}{|l|l|l|l|l|l|l|}
\hline $\begin{array}{l}(\mu \mathrm{m}) \\
\text { Length }\end{array}$ & $\begin{array}{l}(\mu \mathrm{m}) \\
W_{0}^{t i p}\end{array}$ & $\begin{array}{l}\text { ROM } \\
(1 \mathrm{md}) \text { cur }\end{array}$ & $\begin{array}{l}\text { ROM } \\
(2 \mathrm{md}) \\
\text { cur }\end{array}$ & $\begin{array}{l}\text { ROM } \\
(3 \mathrm{md}) \\
\text { cur }\end{array}$ & $\begin{array}{l}\text { ROM } \\
(4 \mathrm{md}) \\
\text { cur }\end{array}$ & $\begin{array}{l}\text { Exp } \\
{[8]}\end{array}$ \\
\hline 400 & 28.7 & 45.18 & 27.26 & 21.52 & 19.78 & 20.25 \\
\hline 500 & 34.28 & 37 & 22.1 & 17.04 & 15.4 & 15.63 \\
\hline 600 & 41.23 & 33.45 & 19.66 & 14.9 & 13.18 & 13.38 \\
\hline
\end{tabular}

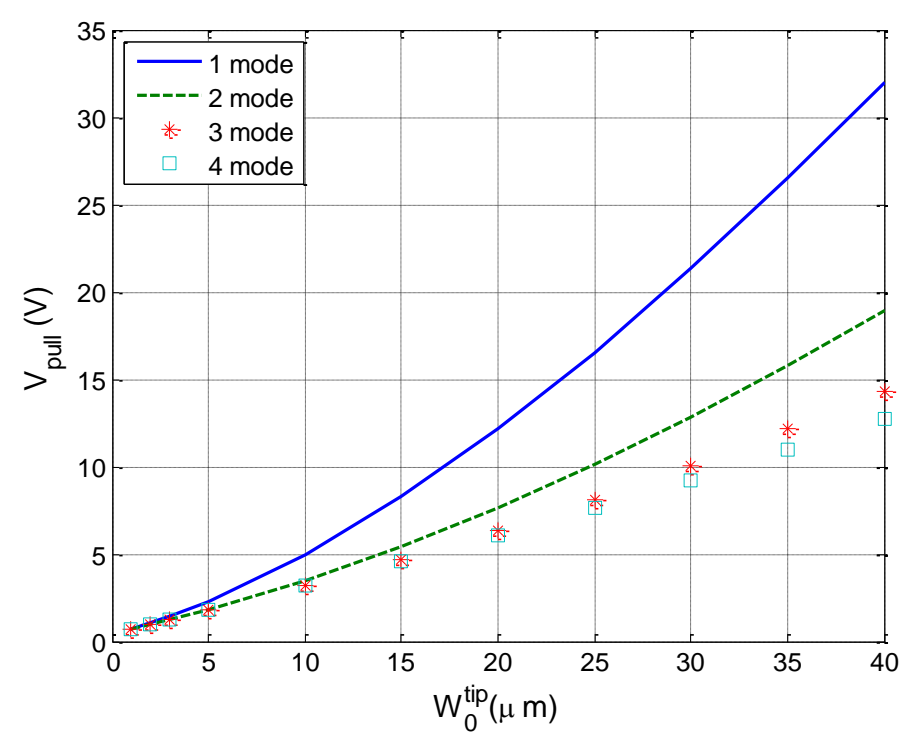

Figure 4: Comparison of the pull-in voltage as calculated using 1-4 modes of the ROM.

\section{SUMMARY AND CONCLUSION}

We presented exact analytical solutions of the electrostatically actuated initially deformed cantilever beam problem. We used a continuous beam model and a single-mode Galerkin technique. We derived simple analytical expressions for two commonly observed deformed beams configurations: the curled and tilted configurations. We compared the results of the derived formulas to experimental results in the literature and numerical results of a multi-mode reduced order model. We found that these formulas yield accurate results for beams of tip deflections of few microns, which is the case commonly encountered in MEMS applications. For largely deformed beams, these formulas cannot be used due to the limitations of the single-mode approximations they are based on. Instead, multi-mode reduced order models need to be utilized. For choosing the curled or titled formulas, one should resort to optical images of the fabricated beams; otherwise formulas both can be used to catch the expected range of pull-in.

Although the focus of this work has been for static analysis, the derived equivalent single-degree-of-freedom models can be an attractive alternative to study the dynamics of these beams compared to the crude lumped-parameter spring-mass-dampers models, which do not account for the initial deformation of the beams, or the cumbersome finite element models. For the generalization of the used technique here for other deformed beams, such as clamped-clamped beams, care should be taken since other factors can cause deformation in these beams, such as the induced axial forces, in addition to the complications coming from mid-plane stretching. 


\section{REFERENCES}

[1] Senturia S D 2001 Microsystem Design (Kluwer Academic Publishers).

[2]Fang W and Wickert J A (1996) Determining mean and gradient residual stresses in thin films using micromachined cantilevers. Journal of Micromechanics and Microengineering. 6:301-309

[3]Younis M I 2011 MEMS Linear and Nonlinear Statics and Dynamics (Springer).

[4]R. Legtenberg, J. Gilbert, S. D. Senturia, " Electrostatic Curved Electrode Actuators," J. Microelectromech. Syst, VOL. 6, NO. 3, SEPTEMBER, pp. 257-265.

[5] Wei L C et al 2002 Analytical modeling for determination of pullin voltage for an electrostatic actuated MEMS cantilever beam Proc. IEEE Int. Conf. on Semiconductor Electronics (Penang, Malaysia) pp 233-8.

[6]M. Lishchynska, N. Cordero, O. Slattery, and C. O’Mahony," Modelling electrostatic behaviour of microcantilevers incorporating residual stress gradient and non-ideal anchors," J. Micromech. Microeng. 15 (2005) S10-S14 doi:10.1088/0960-1317/15/7/002.

[7] $\mathrm{Hu}$ Y-C 2006 "Closed form solutions for the pull-in voltage of micro curled beams subjected to electrostatic loads," J. Micromech. Microeng.16 648-655.

[8] $\mathrm{Hu}$ Y C, and Wei C S 2007 An analytical model considering the fringing fields for calculating the pull-in voltage of micro curled cantilever beams J. Micromech. Microeng. 17 61-67

[9] W-C Chuang, Y-C Hu, C-Y Lee, W-P Shih, P-Z Chang, 2009 Electromechanical behavior of the curled cantilever beam J. Micro/Nanolith. MEMS MOEMS 8033020.

[10] S C Saha, U Hanke, G U Jensen, T Saether, Modelling of Spring Constant and Pull-down Voltage of Non-uniform RF MEMS Cantilever Incorporating Stress Gradient, Sensors \& Transducers Journal, Vol. 98, Issue 11, November 2008, ISSN 1726-5479, pp. 5468.

[11] $\mathrm{Y} \mathrm{J} \mathrm{Hu,} \mathrm{J} \mathrm{Yang,} \mathrm{S}$ Kitipornchai, Pull-in analysis of electrostatically actuated curved micro-beams with large deformation, Smart Mater. Struct. 19 (2010) 065030 (9pp) doi:10.1088/09641726/19/6/065030

[12] Vaishali B.M, Uday V. Wali and Anil V. Nandi, "Analytical Model for Compensating the Curling Effect in MEMS Cantilever Beam," International Journal of Computer Science Issues, Vol. 1, Issue 1, November 2011, ISSN: 1694-0814, pp. 97-100.

[13] S M C Abdulla, H Yagubizade and G J M Krijnen, "Analysis of resonance frequency and pull-in voltages of curled micro-bimorph cantilevers," J. Micromech. Microeng. 22 (2012) 035014 (13pp) doi:10.1088/0960-1317/22/3/035014.

[14] K.-S. Ou, K.-S. Chen, T.-S. Yang, and S.-Y. Lee, "A Novel Semianalytical Approach for Finding Pull-In Voltages of Micro Cantilever Beams Subjected to Electrostatic Loads and Residual Stress Gradients," J. Microelectromech sys, Vol. 20, No. 2, APRIL 2011, 527537.

[15]Younis, M. I., Abdel-Rahman, E. M., and Nayfeh, A. H., “A reduced-order model for electrically actuated microbeam-based MEMS," Journal of Microelectromechanical Systems, Vol. 12, pp. 672--680, 2003.

[16]Nayfeh, A. H., Younis, M. I., and Abdel-Rahman, E. M., "Reduced-order models for MEMS applications," Nonlinear Dynamics, Vol. 41, pp. 211--236, 2005.

[17] Gupta R K 1997 Electrostatic pull-in test structure design for insitu mechanical property measurements of microelectromechanical systems $P h D$ Thesis (Massachusetts Institute of Technology).

[18] Younis, M. I., Miles R., and Jordy D., "Investigation of the response of microstructures under the combined effect of mechanical shock and electrostatic forces," Journal of Micromechanics and Microengineering, Vol. 16, pp. 2463-2474, 2006.

[19] Younis, M. I, Ouakad, H., Alsaleem, F. M., Miles, R., and Cui, W., "Nonlinear Dynamics of MEMS Arches Under Harmonic Electrostatic Actuation," Journal of Microelectromechanical Systems, Vol. 19, Issue 3, pp.647 - 656, 2010. 